\title{
PREFACE
}

\section{Reoperative Surgery}

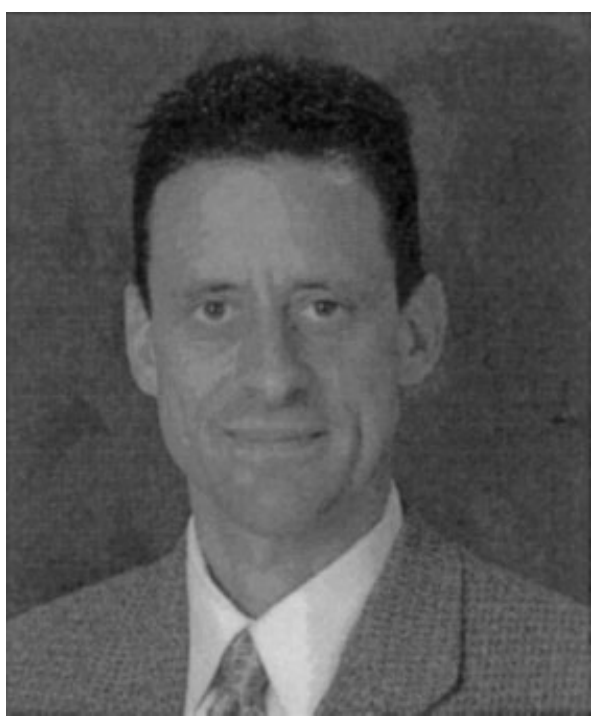

Reoperative surgery is exceedingly common and, depending on the timing and extent of prior operations, may contribute greatly to the complexity and morbidity of the current care of the patient. In this issue of Clinics in Colon and Rectal Surgery, we attempt to look at reoperative surgery from a clinically relevant point of view.

Surprisingly few data on reoperative surgery have existed until recently, and even the data available are inherently biased because of selected populations of patients. Small bowel obstruction is the most recognized complication of prior operation. Drs. Joe Carmichael and Steve Mills from University of California Irvine discuss the various aspects of small bowel obstruction relevant to the practicing clinician, with special emphasis on timing of reoperation and early postoperative small bowel obstruction. The uncommon but potentially devastating complication of intraoperative or postoperative hemorrhage is discussed by Drs. Kerry Hammond and David Margolin from the Ochsner Clinic in New Orleans. They also review the concepts of damage control surgery and abdominal compartment syndrome and bring them into relevance for the practicing colon and rectal surgeon. Drs. Rowena Ramirez and Phil
Fleshner from Cedar Sinai Medical Center in Los Angeles discuss reoperation for inflammatory bowel disease. Sphincter salvage surgery for ulcerative colitis is common, and this article focuses on ileoanal pouch salvage but also reviews reoperation for Crohn's disease.

Anastomotic failure or leak is perhaps the most feared and devastating complication following restorative colorectal surgery. Drs. Zuri Murrell and Michael Stamos review this problem with an eye toward providing the reader with a logical, systematic approach to avoiding and treating this complication. Minimally invasive colectomy in many institutions is becoming more common than laparotomy. Drs. James McCormick and Cliff Simmang are leading proponents of minimally invasive approaches, and they review the critically important topic of whether reoperation following minimally invasive surgery is different from reoperation following open abdominal surgery. Indeed, are the rules different? Dr. Tracey Arnell, from New York Presbyterian, Columbia Medical Center, looks at a related topic from a slightly different point of view. She discusses the role of minimally invasive reoperation following prior open laparotomy. There remain some entities that do not lend themselves to minimally invasive operations or reoperations, and certainly recurrent colorectal cancer is one of these. Arguably, recurrent colorectal cancer requires more planning than any other reoperation, including the decision of whether or not to intervene surgically. Drs. Cesar Santiago and Michael Hellinger of the University of Miami discuss these issues in a cogent fashion.

Drs. Kathryn Galie and Charles Whitlow, from the Ochsner Clinic, cover the topic of enterocutaneous fistula, emphasizing the management of this problem when it occurs in the postoperative period. They provide a very nice roadmap to identifying and managing this problem, including excellent advice on how to succeed. As mentioned earlier, in recent years we have gained the value of good data obtained from randomized prospective trials. Dr. David Beck, from the Ochsner Clinic, who was involved in many of these trials, succinctly details the available data from those trials as they relate to the entity of reoperative surgery. Rounding out this issue of Clinics in Colon and Rectal Surgery is Dr. Tony Senagore's article

\footnotetext{
${ }^{1}$ Division of Colon and Rectal Surgery, Department of Surgery, University of California, Irvine Medical Center, Orange, California. Address for correspondence and reprint requests: Michael J. Stamos, M.D., UCI Medical Center, Division of Colon and Rectal Surgery, Department of Surgery, 333 City Blvd. West, Ste. 850, Orange, CA 92868-3298. E-mail: mstamos@uci.edu.
}

Reoperative Surgery; Guest Editor, Michael J. Stamos, M.D.

Clin Colon Rectal Surg 2006;19:179-180. Copyright (C) 2006 by Thieme Medical Publishers, Inc., 333 Seventh Avenue, New York, NY 10001, USA. Tel: +1(212) 584-4662.

DOI 10.1055/s-2006-956437. ISSN 1531-0043. 
on the economic consequences of reoperative surgery. This is an area that has long been ignored by the surgical community as well as by the payors. As Dr. Senagore aptly points out, more data and more political pressure are needed to "level the playing field" and to make reoperative surgery economically viable, much less profitable, during a period of ever shrinking reimbursements.
It has been an honor and distinct pleasure for me to serve as guest editor of Clinics in Colon and Rectal Surgery and, in particular, to interact with the set of authors who have contributed so greatly and generously.

Michael J. Stamos, M.D. ${ }^{1}$

Guest Editor 